\title{
Las emociones y la privación de libertad como tratamiento. Acercamiento al control de la ira en el INISA
}

\author{
Emotions and imprisonment as treatment. \\ Approach to "anger management" at INISA
}

\section{Luis Ignacio Estrade Weikert*}

\footnotetext{
* Licenciado en Ciencias de la Educación (Facultad de Humanidades y Ciencias de la Educación, UdelaR). Estudiante de la Licenciatura en Ciencia Política (Facultad de Ciencias Sociales, UdelaR). Docente de ajedrez (ANEP) y coordinador de un centro educativo de educación de jóvenes y adultos. Participa en las líneas de investigación «Emociones, narraciones y ciudadanía» y «Escuela y cárcel: igualdad y diferencia» (UdelaR). Investiga temas de ajedrez y pedagogía en el Uruguay.

凹· weikert.19@gmail.com https://orcid.org/0000-00026385-4842
}

RECIBIDO: 30.9 .2019

ACEPTAD0: 4.11 .2019

\section{Resumen}

La investigación, llevada a cabo en 2018, se propuso abordar aspectos vinculados a la educación emocional y aportar una reflexión transversal sobre algunas de las características que la conforman. Precisamente, a través de este texto alentamos una mirada examinadora de ciertos elementos que estructuran las políticas educativas sobre las emociones, enfocándonos sobre la educabilidad de estas, la narrativa y la reflexión, en un contexto de privación de libertad adolescente que puede reproducir, legitimar y naturalizar ciertos lugares de imposibilidad. Asimismo, anotamos elementos para identificar las relaciones y diferencias entre las teorías de las emociones y las políticas implementadas. Palabras clave: psicología, educación, sanción penal, joven, Uruguay.

\section{Abstract}

The research, carried out in 2018, aimed to address some aspects of emotional education and to provide a cross-sectional analysis on some of its features. This text specifically encourages a scrutinizing approach to some of the structuring elements of emotional educational policies, focusing on their educability, narrative and reflection, in a context of imprisonment of adolescents 
that can reproduce, legitimize and naturalize certain places of impossibility. Likewise, elements to identify the relationships and differences between the theories of emotions and the policies implemented were defined.

Keywords: psychology, education, penal sanctions, youth, Uruguay.

\section{Introducción}

El presente trabajo surge de la preocupación por dar cuenta de las políticas de educación emocional en Uruguay, que ponen en juego aspectos sustantivos de la convivencia democrática. Nos proponemos indagar específicamente en los componentes del Programa de Tratamiento Grupal Control de la Ira $^{1}$ (en adelante, PTGCI), desarrollado en el Instituto Nacional de Inclusión Social Adolescente (en adelante, INISA) durante el año 2016, con base en 24 encuentros de trabajo grupal, denominados sesiones, para las personas que se encontraban allí en situación de privación de libertad (Instituto Nacional de Inclusión Social Adolescente [INISA], 2016a). Dicho programa emana de una adecuación uruguaya del Manual del prisionero para controlar la ira, escrito e implementado en cárceles canadienses de adultos por el psicólogo Murray Cullen (1992). Nos apoyamos teóricamente en una articulación entre las nociones de educación emocional, metaemociones y autorreflexión (Modzelewski, 2017). A la luz de autores como Frankfurt (2006) y Nussbaum (1997), trabajaremos la posibilidad de la educación de las emociones y la narración como instrumento posibilitador.

En el caso de los espacios de encierro, el contexto adquiere un lugar central en el análisis de lo educativo. Sobre esa base, llevamos adelante una lectura de lo propuesto por el documento del programa y de la evaluación institucional de su aplicación en los centros de reclusión, apuntando al análisis de las debilidades que presenta a la luz del marco teórico con el que trabajamos. No abordamos aquí los resultados empíricos - lo cual requiere permisos institucionales especiales y un marco metodológico atento a las limitaciones que presenta dicho contexto-, aunque entendemos que sería pertinente y relevante analizarlos en una investigación posterior.

El trabajo se desarrolla en dos partes. En primer lugar, nos proponemos hacer un abordaje descriptivo del documento a analizar, buscando dar cuenta de la caracterización de los sujetos, los objetivos y la metodología. Seguidamente, abordaremos la educación de las emociones como postura filosófica necesaria para el encuentro con el otro. Buscamos estudiar la convivencia desde un punto de vista emocional, discutiendo las consideraciones del yo, del otro y de lo común. Hemos priorizado el estudio de lo 
simbólico en la construcción de la otredad y del uno mismo en un contexto de privación de libertad de los aprendientes, así como los márgenes de la libertad individual en una comunidad moral, y su vínculo con el conflicto. Tomaremos lo que Frankfurt (2006) denomina autoevaluación reflexiva, vinculándolo con el deseo y la voluntad. Las construcciones de sentido sobre la educación de las emociones se apoyan en la viabilidad de alcanzar grados de reflexividad constantes, a pesar de los contextos en que se está inmerso.

Por último, estudiaremos el informe posterior de la experiencia, realizado por el mismo equipo de psicólogos que implementó el programa, en el cual retoma algunos conceptos trabajados anteriormente en cuanto a la influencia del entorno, los cometidos institucionales reales del INISA, y sus puntos de acuerdo y tensión con el marco teórico mencionado. A la luz de dicho informe encontramos, por un lado, los aspectos formales y teóricos del protocolo; por otro, los reales, en el sentido de su aplicación, de sus avatares institucionales y de su anclaje subjetivo.

\section{Marco del programa}

El PTGCI (INISA, 2016a) debe su nombre y los términos generales del contenido al libro Enjaula tu furia, un manual para prisioneros abocado a ayudarlos a controlar su ira, basado en el Programa de Control del Enojo, del psicólogo especializado en trabajo penitenciario Murray Cullen (1992), aplicado en algunas cárceles de Canadá. El INISA (2016a), en el marco del «trabajo referido a la salud mental» de las personas privadas de libertad en su ámbito, delegó al equipo de psicólogas del Programa Técnico Psicosocial de dicho organismo la responsabilidad de diseñar e implementar un programa similar, con la particularidad de abocarlo al trabajo colectivo de los adolescentes, mientras que el de Cullen (1992) es para adultos e individual. El del INISA fue diseñado para ejecutarse con el total de las personas que cumplían penas de privación de libertad en el organismo, en todas las unidades, tanto masculinas como femeninas.

El objetivo del programa, que se desarrolló a lo largo de un semestre para cada uno de los centros de reclusión, es «ayudar a los adolescentes a reconocer sus sentimientos de enojo, sus causas, y a enfrentarse a este sentimiento de una manera nueva, una manera responsable que probablemente no fue la forma en que él aprendió en el pasado» (INISA, 2016a, p. 13).

El programa se presenta, a lo largo de 51 páginas, con un análisis del perfil de los adolescentes, una fundamentación de la propuesta de trabajo grupal, los objetivos, la población objetivo, la fundamentación para la aplicación del programa, un encuadre técnico para la implementación, una consigna y una metodología de intervención, una caracterización de las habilidades terapéuticas necesarias, y luego un amplio desarrollo 
de la propuesta dividido en 24 sesiones, con una planificación exhaustiva, ordenada diacrónicamente, y de las posibles contingencias de la implementación.

\section{Propuesta de intervención}

Si bien el trabajo de Cullen (1992) es de enfoque individual, en el INISA se consideró la importancia de lo colectivo, de que el espacio grupal habilitara ámbitos de socialización y subjetivación, y que la posibilidad de que esto se replanteara en la mirada de la institución pudiera ser factor preponderante de cambio. En espacios colectivos los adolescentes ponen en cuestión sus roles dialogando con los demás, lo que implica canalizar el encuentro con los otros para potenciar el intercambio reflexivo: «La posibilidad de dar la palabra y que esta sea escuchada favorece el desarrollo y ejercicio de habilidades yoicas y sociales permitiendo el reconocimiento del mundo interno, el cuidado por sí mismo, y por el otro» (INISA, 2016a, p. 11).

El programa proponía «talleres grupales de tratamiento», con el objetivo de estimular en los adolescentes el desarrollo de procesos psíquicos que favorecieran el autoconocimiento y la conciencia de sí mismos. Buscó aportar en la modificación de las estrategias de abordaje de los problemas cotidianos con vistas a obtener mejores resultados.

A través de la intervención grupal, se pretendió que los adolescentes alcanzaran los siguientes objetivos generales:

Reconocer sus sentimientos de enojo, analizar sus causas, para así poder procesarlos adecuadamente, y enfrentarse a ellos de una manera responsable. Lograr controlar estos sentimientos de enojo, para evitar acciones dañinas a sí mismos o a los otros. Estimular el desarrollo de la conciencia crítica, de modo de lograr alcanzar una autonomía en su evolución. Ampliar su repertorio de experiencias: sensoriales, afectivas, cognitivas, simbólicas, culturales, apuntando a un proceso gradual de integración y organización de las mismas (INISA, 2016a, p. 12).

La consigna disparadora que se desprende de los objetivos es: «Nadie puede hacer que tú te enojes, nadie puede hacerte sentir nada. Tú eres el que decide cómo es que tú te quieres sentir» (INISA, 2016a, p. 12). Se planteó una dinámica de trabajo para grupos integrados por diez adolescentes. En cada centro de detención se conformó una Junta de Tratamiento del Programa (INISA, 2016a), que se encargaría de distribuir los grupos de adolescentes con los que se trabaja, según las variables que cada junta consideró que condicionaban la dinámica del grupo. Como criterio general, se estableció que los coordinadores trabajaran focalizadamente $-y$, si fuera necesario, de forma paralela al grupo- con aquellos adolescentes que presentaban problemas de integración y que 
habían experimentado dificultades en el proceso de aprendizaje y reflexión colectiva, para no alterar el normal transcurso del programa. Se estipuló la importancia de permitir circular la palabra, que todos pudieran opinar, y que si fuera necesario se facilitara el diálogo a través de preguntas.

\section{Educación emocional}

\section{Definición y objetivos de la educación emocional}

Daniel Goleman (1995), uno de los más importantes divulgadores de las últimas investigaciones sobre inteligencia emocional y sus posibilidades de educación, señala en el siguiente párrafo los elementos principales que deben conformar una adecuada educación emocional:

Si el desarrollo del carácter constituye uno de los fundamentos de las sociedades democráticas, la inteligencia emocional es uno de los armazones básicos del carácter. La piedra de toque del carácter es la autodisciplina — la vida virtuosaque, como han señalado tantos filósofos desde Aristóteles, se basa en el autocontrol. Otro elemento fundamental del carácter es la capacidad de motivarse y guiarse uno mismo, ya sea para hacer los deberes, terminar un trabajo o levantarse cada mañana. Y, como ya hemos visto antes, la capacidad de demorar la gratificación y de controlar y canalizar los impulsos constituye otra habilidad emocional fundamental a la que antiguamente se llamó voluntad. (Goleman, 1995, p. 179)

Contamos en estas pocas palabras con las bases de la educación emocional según es llevada a cabo mayoritariamente en la actualidad. Y en estas palabras se destaca, en primer lugar, la mención de las sociedades democráticas.

Es que, como baluarte de una ciudadanía política activa, la sociedad se debe plantear como una consideración detallada de las singularidades en juego. La igualdad surge a partir de las subjetividades, de lo diferente, y la justicia que se persigue no puede ser ajena a esto. Este enfoque busca estudiar cómo una sociedad garantiza que la ciudadanía se ejerza mediante un rol activo. Atender a las formas en que se potencia esa proactividad requiere, sin duda, atender a las emociones, entendidas como parte central de la toma de decisiones de los sujetos.

Por lo tanto, es posible afirmar que, para que una democracia se precie de ser tal, sus integrantes la deben sostener mediante la iniciativa, el agenciamiento colectivo, habilitado de forma horizontal y circulante. La lucha contra las injusticias tiene allí su 
punto de inicio, y aquellas dinámicas sociales que infligen gran dolor a la población serán objeto de cuestionamientos y reformas a partir de esta lucha. En la persecución de tales objetivos, la educación de las emociones no ha sido suficientemente tenida en cuenta, y esta es una de las razones por las que la población del INISA, como muchas otras, encuentra falencias considerables en el ejercicio de sus derechos más básicos.

Sin embargo, es preciso comprender que se requiere cierta longevidad para que los programas tengan incidencia real en la vida de los sujetos. Los aprendizajes se alcanzan con la constancia de los cursos en el correr de los años, de modo de ir fortaleciendo ciertas habilidades emocionales estratégicas. Las situaciones de desborde y agresividad se caracterizan por el exceso y la irracionalidad, y las formas de ir controlando eso se deberían trabajar prolongadamente, apuntando a resultados a mediano y largo plazo.

\section{Las emociones en la cárcel}

Como seres humanos con capacidad de reflexión abstracta, contamos con la sabiduría de integrar en nuestras valoraciones a personas, objetos y espacios que no tenemos a nuestro alcance ni visual ni tangiblemente. Ese patrimonio humano muchas veces agudiza, en privación de libertad, ciertas emociones de extrañamiento, de soledad o de falta. Sin embargo, es fundamental germinarlo a los efectos de que los sujetos cultiven sus procesos de razonamiento abstracto, como condición para un estadio cognitivo y emocional desarrollado. Reflexionar sobre la privación de libertad conlleva retomar la búsqueda de justicia como génesis de la educación de las emociones. Es pensar al otro como sujeto decisor y no como objeto de tutela, y es brindarle una educación acorde para que tome decisiones acertadas. Refiere a proveer las garantías para que todos los ciudadanos puedan recibir y brindar un trato igualitario a sus pares, no solo como receptores de decisiones estatales de gobierno, sino como gestores de justicia en lo interpersonal. Estos objetivos forman parte, junto con otras propuestas filosóficas y políticas, del conocido enfoque de las capacidades presentado por Martha Nussbaum y Amartya Sen (1996). Es prioritario atender las motivaciones de las personas y sus aspiraciones a realizarse en tal o cual faceta de la vida, devenidas identidades diferenciales en el encuentro con los otros, y esto señala el importante valor de una educación emocional.

Aunque las teorías clásicas de la pena arguyen que la privación de libertad es un espacio para que el sujeto reflexione en torno al delito cometido, la cárcel no convoca per se a la reflexividad, como tampoco lo hace el aislamiento. Las lógicas carcelarias delimitan reglas de juego para las personas que, independientemente de las formas en que se vinculan en el exterior, las llevan a adoptar distintos roles en plan de disminuir los dolores del encarcelamiento (Sykes, 1958). En el caso del INISA - y en sintonía con la 
tendencia latinoamericana-, los criterios de comportamiento tienden a ser los de la enajenación y la humillación simbólica de los adolescentes, lo que deriva en la búsqueda de la ventaja relativa, la conveniencia individual y la supresión del otro, en un contexto de mortificación del yo (Goffman, 1961/2001) donde las variadas dimensiones de la libertad individual quedan suprimidas. Este fenómeno ha sido señalado como preocupante por recientes investigaciones en la materia (Casa Bertolt Brecht, 2015; Institución Nacional de Derechos Humanos y Defensoría del Pueblo [INDDHH], 2018). Se fortalecen comportamientos instintivos, predominantes entre aquellos promovidos por el entorno de la cárcel (Zaffaroni, 1998), en que la voluntad verdadera de los sujetos queda relegada. Esto coincide con la definición de Frankfurt de agente inconsciente:

No hay nada en el concepto de agente inconsciente que implique que este no pueda razonar o deliberar acerca de cómo hacer lo que quiere hacer. Lo que distingue al agente inconsciente racional de otros agentes racionales es que no le preocupa la conveniencia de sus deseos. Hace caso omiso de la pregunta acerca de cuál ha de ser su voluntad. No solo sigue cualquier proceder que se vea más fuertemente inclinado a seguir, sino que no le importa cuál de sus inclinaciones es más fuerte. (Frankfurt, 2006, p. 33)

Sin embargo, nos interesa recabar posturas como la de Nussbaum (1997), que toman distancia del concepto de inconciencia para hacer hincapié en lo que entienden es la cualidad sustantiva de los seres humanos: la posibilidad de deliberación basada en la lógica. Desde esta perspectiva, la disyuntiva, la comparación y la deliberación de las emociones hacen a los sujetos en cuanto tales y, por lo tanto, no hay contexto habitacional o emocional que pueda inhibirlas. Independientemente del grado de completitud que impliquen, todos contamos con dichas características. Las emociones humanas se distinguen por ser poseedoras de sentido lógico. Para dar cuenta de ello, Nussbaum (1997) explica cómo los seres humanos, a diferencia de otros animales, adoptan emociones desacertadas y conductas también desacertadas, o carentes de sentido lógico, pero que eso despierta la deliberación valorativa del sujeto sobre sí mismo, de modo que de ese replanteamiento derivan criterios racionales de toma de decisiones. Esto no implica un cambio automático en las conductas, y menos en las emociones, pero permite el primer paso hacia el cambio que es el cuestionamiento de lo establecido. El lenguaje tiene un papel importante en el cuestionamiento, ya que es lo que nos permite significarnos y nominar a las emociones. Los programas orientados a la educación de las emociones cumplirían con la función de incentivar que dicho cuestionamiento de lo establecido, el replanteamiento de uno mismo, derive en resultados emocionales y comportamentales favorables (Modzelewski, 2017). 


\section{Bases ontológicas y posibilidades de la educación emocional}

Al referirnos a lo emocional como objeto de estudio, nos encontramos con miradas disimiles respecto al anclaje corporal y filosófico de las emociones. De ahí los alcances y limitaciones de los programas de educación emocional.

Una de las disyuntivas principales reside en la conceptualización de las emociones en relación con diversos componentes de lo humano que las determinan desde lo fisiológico como objeto de educabilidad o no. Por una parte, están aquellos que las consideran como una característica humana ajena a la razón, es decir, que puede ser interpretada por la neurociencia, la psicología cognitivista y las ciencias de la salud, pero que responde a conductas ajenas a la intervención externa y, por tanto, a lo educativo.

También, desde otro lado, están las ramas de la psicología que se enfocan en los componentes impulsivos del comportamiento humano, aquellas que adjudican intencionalidad a partir de impulsos o pulsiones (Freud, 1930) ajenas a la voluntad. Es ilustrativo de ello el análisis de Modzelewski (2016), quien encuentra cuatro vertientes en las que pueden clasificarse las posturas respecto a las emociones, tanto a lo largo de la historia de la filosofía como en los estudios contemporáneos, según las entiendan como: a) íntegramente cognitivas, b) predominantemente cognitivas con una importante intervención de aspectos fisiológicos, c) predominantemente fisiológicas con un importante componente cognitivo y d) íntegramente fisiológicas. Todas ellas, sin embargo, dan lugar a su educación.

Nuestra perspectiva para analizar la educación de las emociones planteada en el INISA considera también un hecho la educabilidad de las emociones, y coincidimos con Modzelewski (2017) en cuanto a la importancia de ponerlas en palabras y estudiarlas para buscar estadios emocionales deseables. El sujeto se erige como un ser autogestivo, de modo de asumir la responsabilidad de sí. Por lo tanto, las corrientes estudiadas aquí se presentan alejadas de un determinismo neurológico.

Según los antiguos estoicos, uno de los componentes de lo humano imprescindibles para que las emociones tengan lugar es la noción de juicio, que da cuerpo a la reflexión. A esta postura adhiere Martha Nussbaum $(1993,2005)$ para fundamentar la posibilidad de educar las emociones. La racionalidad subyacente a las emociones las hace objeto de educación, porque las emociones son regulables al serlo los juicios que las generan. Es necesario, ante todo, que esos juicios sean leídos como algo a mejorar, discriminando, enjuiciándolos a ellos. La voluntad de replantearse el statu quo emocional es el motor para que las emociones sean educadas. Solo así se pueden esperar frutos de un programa educativo para las emociones, y aquellos que no logren sembrar la duda en los sujetos serán inocuos.

La libertad que uno posee, la que ejerce con cada acción, es más profunda que la libertad de tomar decisiones. La libertad de la voluntad no se limita a hacer lo que uno 
quiere. Tampoco es condición necesaria: la privación de la libertad de circulación no debería limitar, en teoría, la libertad de la voluntad.

Cuando un agente es consciente de que hay ciertas cosas que no es libre de hacer, ello, sin dudas, afecta sus deseos y limita el rango de las elecciones que puede hacer. Pero supongamos que alguien, sin saberlo, ha perdido o ha sido privado de su libertad de acción. Aunque ya no tiene la libertad de hacer lo que quiere hacer, es probable que su voluntad siga siendo tan libre como lo era anteriormente. (Frankfurt, 2006, p. 37)

La pregunta de si la voluntad de una persona es libre tiene que ver con los deseos en sí mismos, pero además con el entorno, con los límites espaciales. No obstante, el mismo autor indica: «La pregunta por la libertad de su voluntad no tiene que ver con la relación entre lo que hace y lo que quiere hacer. Más bien tiene que ver con sus deseos en sí mismos» (Frankfurt, 2006, p. 38). Se trata de querer lo que uno quiere querer, y en esto consiste la autorreflexión, aunque hay una gran diferencia con llevarlo a los hechos. A su vez, cabría preguntarse qué pasa con la frustración cuando uno no puede querer lo que quiere querer, y qué pasa cuando uno no puede llevar a la práctica lo que quiere.

Ejemplifiquemos. Un adolescente privado de libertad en el INISA se encuentra en una celda para dos personas conviviendo con tres más. Dos duermen en las camas y dos en colchones en el piso. Al adolescente le toca estar al lado del wáter y se despierta frecuentemente en las noches por el mal olor. Anhela que llegue un día en que todos tengan mejores condiciones de vida y no necesiten pelearse a fuerza de cuchillos por quién duerme al lado del wáter. Esta vez le tocó a él, y la infección de la herida del brazo no se va, pero sabe que luego vendrán otros. Son espacios de alta rotatividad. Después de una tarde de reflexión con uno de los compañeros de celda, estuvieron de acuerdo, en voz baja, en que la pelea con arma blanca no era la mejor forma de dirimir quién dormía dónde. Pensaron en plantearlo en la celda, pero las represalias iban a ser grandes y toda la cárcel -funcionarios, policías e internos- les tomaría el pelo y haría de ellos verdaderos objetos de juego. La voluntad reflexiva del adolescente se impuso frente a su deseo de pelear de nuevo para ganar un mejor lugar, pero las condiciones vinculares en las que vive continúan siendo exactamente las mismas: sabe que en breve le va a tocar pelear de nuevo, y no ha podido externalizar su voluntad para que el suplicio no sea aún mayor. Decide, también reflexivamente, no hacer eco de su voluntad para evitar los daños que pueda generarle. La pragmática irreflexiva de la mayoría se ganó a otra persona. 


\section{Voluntad, sujetos y cambios}

Para autores como Frankfurt (2006), el foco de la discusión se encuentra en la definición filosófica - no antropológica- que hace al ser humano una especie singular. Las personas, tomando al autor, se distinguen de otras especies por su voluntad. Todos los animales toman decisiones y tienen deseos que pueden ser asociados con las pulsiones instintivas. Los humanos se distinguen en la medida en que obedecen a una voluntad individual, singular, que los hace diferentes respecto de otros humanos en sus decisiones. Además de desear y tomar decisiones, los deseos de segundo orden hacen que - a diferencia de los de primer orden, que emanan de los impulsos - los humanos deseemos desear, prefiramos optar por tener unos deseos y no otros. Tenemos la potestad de plantearnos querer ser diferentes de lo que somos, de evaluarnos reflexivamente a la luz de nuestros deseos para con nosotros mismos, y de actuar en consecuencia. A ese desempeño consecuente el autor lo llama volición de segundo orden (Frankfurt, 2006).

El deseo para Frankfurt (2006) tiene que ver con la voluntad. Identificar la voluntad significa identificar el deseo en el cual se basa cierto acto, o del cual emana la inacción. Desde esta perspectiva, la voluntad se distingue de la intención. La intención es una manifestación de deseo, no necesariamente algo más que eso, pero la voluntad es el deseo de que el deseo anhelado se convierta en voluntad. El autor sostiene que para considerar a alguien una persona, es necesario que esta experimente voliciones de segundo orden, no solo deseos.

El anhelo de poseer una emoción no se rige por el placer que esta puede llegar a generar. La vida que uno aspira a vivir se encuentra en juego. Para trabajar el sujeto que uno desea ser es necesario un tiempo de reflexión prolongado y las habilidades cognitivas necesarias para replantearse existencialmente, en busca del estilo de vida que se está dispuesto a vivir. Esa reflexión sobre uno mismo requiere mucha solidez emocional, evaluación responsable sobre uno mismo basada en los anhelos mencionados.

Ahora, rara vez las emociones son momentáneas; en casi todos los casos están insertas en procesos diacrónicos extendidos en el tiempo, que tienen un pasado y pronostican un futuro. Esta característica facilita el análisis que se puede hacer de ellas, al tiempo que da cuenta de la necesidad de contar con criterios establecidos para canalizarlas, de modo de alcanzar una unidad de sentido que Robert Solomon (2007) denomina integridad emocional.

Identitariamente, los sujetos se erigen a partir de la integridad. La integridad es la puesta en juego de los principios que hacen a la identidad de uno. Y quienes contradicen esos principios no son agentes externos, sino justamente deseos de primer orden. Una forma posible de medir la integridad de las personas es la solidez con que uno hace valer esos principios frente a otros deseos y los transforma en hechos concretos: «Los 
principios determinan obligaciones incondicionales cuyo incumplimiento significaría la pérdida de nuestra identidad» (Modzelewski, 2017, p. 16).

Aquellos que no lo viven, a quienes no les importa su voluntad, son agentes inconscientes, carentes de voluntad. Los deseos de segundo orden, aquellos atravesados por la voluntad, expresan lo que Frankfurt (2006) dio en llamar autoevaluación reflexiva. Si las personas no experimentaran dicha característica, carecerían de autonomía y quedarían sujetas a sus emociones de forma irracional e instintiva. Así, los deseos por sí solos no representan una actitud proactiva de cambio. El deseo de segundo orden proveniente de una autorreflexión, y su expresión en una volición de segundo orden, emanan con frecuencia de una emoción de arrepentimiento, disgusto u otros.

Los deseos de segundo orden, los deseos de desear otras cosas, pueden ser inducidos por diferentes factores. En el caso del INISA, la génesis del espacio es un programa institucional cuyo primer cometido es problematizar la zona de confort de los asistentes, para que autorreflexionen y se planteen otras formas posibles de vincularse. Así, vemos que los deseos de segundo orden pueden estar provocados mayormente por sentimientos de culpa originados por situaciones de exceso que derivan en violencia indebida hacia terceros o hacia uno mismo.

Por otro lado, las voliciones de segundo orden, como acontecimiento, son altamente dificultosas en contexto de privación de libertad debido a las resistencias emocionales de las personas. A modo de ejemplo, una madre puede verse tomar malas decisiones respecto al cuidado de su hijo debido al amor que este le despierta. Dichas decisiones pueden ser de defenderlo en situaciones en que no tiene la razón, o dejarle pasar ciertos errores para que no se sienta atacado, o comprarle algún objeto innecesario simplemente para despertarle una sonrisa. Esas acciones surgen a partir de una autorreflexión conscientemente meditada, pero entran en tensión con otros criterios de evaluación de las emociones. Un ejemplo frecuente en privación de libertad, por las condiciones habitacionales y materiales con que se vive, es el sentimiento de envidia para con el de al lado y su visita - la visita de la familia, que le lleva comida y bienes básicos de higiene al adolescente-. Es un sentimiento que parece imposible de extirpar, que es realmente genuino, pero que no se desea. Las voliciones de segundo orden se ponen en cuestión frente a la tensión con las emociones viscerales, intensas, como la envidia o el amor.

\section{De la escritura y pensarse a sí mismo}

En la educación de las emociones es clave despertar el deseo y la necesidad de cambio en el sujeto aprendiente. Para él, pensarse a sí mismo implica juzgarse. Para que esto suceda más allá de los acontecimientos de reflexión, es crucial poner en palabras 
que permanezcan, sobre las cuales volver: escribir. La autorreflexión cuenta con la narración, en un lenguaje, como pilar estratégico. Las palabras enriquecen el análisis y las alternativas, les dan más precisión y más vías posibles de solución. Más aún: en la medida en que se desarrolle el alfabeto enfocado hacia lo emocional, se profundizará la reflexión sobre uno mismo. Esto es posible en el encuentro con otros con los cuales entenderse. Uno, al escuchar al otro y reconocer sus emociones, ejercita su inteligencia emocional. Se da el espacio a deseos de segundo orden, a reflexiones sobre qué se quiere desear, qué se anhela, identificarse con emociones a reivindicar para sí. A estos efectos, los sujetos discernimos, comparamos, evaluamos diferentes emociones y nos posicionamos. Juega un rol protagónico la razón, al establecer deseos y silogismos.

A estas reflexiones emocionales acerca de las emociones — que son, en otras palabras, autorreflexión (la reflexión sobre las emociones de uno mismo) - las llamamos metaemociones. Son las que inducen al cambio y habilitan a pensar a las emociones en términos educativos. A los adolescentes privados de libertad, sujetos emocionalmente educables, se les debería incentivar sus metaemociones (Modzelewski, 2016, 2017).

A estos efectos, la narración adquiere un rol protagónico (Nussbaum, 1997). Es parte sustantiva de la autorreflexión. A esta se apunta con una fuerte impronta en el programa analizado. Implica revelar, antes de la implementación del programa, si todos los participantes poseen conocimiento básico de lectoescritura. A quienes no lo poseen deberían proveérseles las herramientas necesarias. Además, cultivarlos en el uso de la lengua para nutrir el vocabulario con que nominan las emociones. Dígalo con mímica, obras de teatro que representan situaciones, la síntesis común de los trabajos colectivos entran en la narrativa como forma de comunicación con otros. Gracias a estas herramientas comunicacionales uno puede expresar sus emociones y plasmar las estructuras que las erigen (Modzelewski, 2016). El componente narrativo de las emociones (Nussbaum, 1997) nos permite rastrear la génesis de estas, profundizar los hallazgos y generar cambios concretos en la forma de externalizarlos.

La educación de las emociones, más que máximas en torno a qué emociones desear o imperativos morales sobre unas u otras, consiste en la incorporación de dichas técnicas reflexivas acerca de las emociones. Se trata de ejercitar dinámicas de autorreflexión de modo que formen parte consciente de nuestras decisiones. Acordamos con Modzelewski en la preponderancia adjudicada al ejercicio de la autorreflexión, así como en que esta se asimila a lo que Paulo Freire denomina concientización, que consiste en el movimiento hacia una conciencia crítica, característica de los regímenes auténticamente democráticos (Modzelewski, 2016).

Desde un punto de vista hegeliano (Hegel, 1987), el conocimiento de la propia conciencia solo puede ser alcanzado en el encuentro con otro, con otra conciencia. El análisis a partir de la intersubjetividad permite a uno objetivarse desde la alteridad. La interacción es la forma con la cual uno reflexiona sobre sí mismo. El PTGCI (INISA, 2016a) 
trabaja, en teoría, en ese sentido: grupos de pares que no avasallen la intimidad pero que habiliten espacios de puesta en común, de nominarse y evaluarse en colectivo. La apertura a la revisión a partir de lo colectivo y la acertada identificación de los problemas hacen a los sujetos personas críticas, capaces de repensarse responsablemente.

La narración es, asimismo, a los efectos prácticos, una gran herramienta. Se ancla en la lengua, en estructuras simbólicas que nos permiten interpretar el mundo y entendernos con nuestros pares. En la medida en que los sistemas de símbolos se complejizan, se hacen más precisos. Pueden definir con más certeza el fenómeno al que se alude. El desarrollo entonces de esta debe ir fuertemente de la mano con la educación de las emociones. La nuevalengua planteada por Orwell en su conocido libro 1984, o la persecución de los libros en Fahrenheit 451, de Ray Bradbury, nos hablan de la necesidad de complejizar, de la defensa del pluralismo de la lengua y la precisión lingüística. En las cárceles hay una gran batalla para dar en ese aspecto.

Asimismo, no se puede pasar por alto que las personas externalizan las emociones de diversas formas, que muchas veces trascienden lo narrativo. Nominar, sin embargo, permite identificar problemas. 0 , más bien, problematizar los problemas. Toda expresión emocional o emoción molesta podrá ser objeto de intervención de la voluntad del sujeto en la medida en que se la nomine como emoción y, sobre todo, como problema. Con ello nos permitimos reconocer las suposiciones que las fundan.

\section{Lectura institucional del perfil de los sujetos}

El «Perfil de los adolescentes infractores» desarrollado en el documento (INISA, 2016a) plantea que estos presentan deficiencias intelectuales, afectivas, culturales, laborales y de salud que es necesario tener presentes. El ausentismo escolar, con su consiguiente déficit académico, el déficit de atención, la falta de perseverancia y de disciplina, el no contar con hábitos de trabajo, el estilo de vida «escasamente pro-social» (INISA, 2016a, p. 7), la participación en subculturas marginales relacionadas con la delincuencia, drogas y violencia, la permeabilidad a la influencia de elementos culturales dominantes tales como televisión, publicidad y consumismo, un ocio de escaso aporte para el desarrollo personal, una baja o disfuncional autoestima, una escasa tolerancia a la frustración, la dificultad para la empatía, para asumir normas, y para comunicarse, son algunos de los fenómenos envolventes.

El trabajo (INISA, 2016a) caracteriza ciertos retrasos evolutivos de los adolescentes en términos cognitivos. Dichos retrasos se plantean como la carencia de destrezas consideradas esenciales para la adaptación social. Algunos de los déficits encontrados son la impulsividad, la externalidad («en cuanto no se sienten responsables de lo que les sucede»), el razonamiento concreto, en contraposición al abstracto, la rigidez 
cognitiva, la intolerancia, la resolución cognitiva de problemas interpersonales («aspecto relacionado con la falta de capacidad para detectar y definir un problema, encontrar posibles soluciones, encontrar posibles causas y pensar soluciones alternativas y sus consecuencias»), el egocentrismo, descrito en forma llamativa como «ven el mundo desde su propio punto de vista», los «valores ausentes o precarios» y una insuficiente capacidad crítica (INISA, 2016a, p. 8).

Asimismo, el trabajo menciona el DSM-IV —el Manual diagnóstico y estadístico de los trastornos mentales de la Asociación Estadounidense de Psiquiatría一, según el cual algunas características que se repiten en las trayectorias de vida de los adolescentes los condicionan al desarrollo de un «trastorno disocial» (INISA, 2016a, p. 9). Algunas de ellas son El abandono de los padres en la infancia, el temperamento difícil de los niños y adolescentes, las prácticas educativas «incoherentes con disciplina dura» [sic] (INISA, 2016a, p. 9), los abusos físicos o sexuales, el haber vivido los primeros años de vida en instituciones, los cambios frecuentes de cuidadores, las familias numerosas, la asociación a un grupo de compañeros delincuentes y ciertos tipos de psicopatología familiar. El análisis del perfil de los adolescentes finaliza con las siguientes consideraciones:

[...] conseguir que los adolescentes infractores tomen las riendas de su propia vida de manera responsable es tarea fundamental y para ello es preciso llevar a cabo una intervención que posibilite que los sujetos puedan compensar las fragilidades que han ido acumulándose en su desarrollo personal, a fin de reintegrarlos en la sociedad como ciudadanos libres, formados, responsables y comprometidos. (INISA, 2016a, pp. 9-10)

El análisis psicosociológico que se hace (INISA, 2016a, pp. 7-9) no define explícitamente los conceptos utilizados. Esta caracterización además provocó, según se verá, que a la hora de implementar el programa en cada uno de los centros se priorizaran aspectos concernientes a la seguridad frente a los educativos.

\section{Evaluación del informe}

En el PTGCI (INISA, 2016a) se abordan emociones que son canalizadas en sociedad, que tienen manifestaciones externas y se expresan con impacto en quienes nos rodean. Estas son, fundamentalmente, el enojo y la ira, conceptos entrelazados sobre los cuales no es posible hacer análisis separados. Como indica Modzelewski (2017, p. 6): «La ira o la cólera, por ejemplo, no pueden llamarse tales si no vienen acompañadas de su expresión violenta. Si no lo hicieran, podrían llamarse simplemente enojo o enfado, pero nunca ira o cólera». 
La definición de esta palabra nos convoca especialmente, por ser la que da nombre al programa. Si bien notamos que en el cuerpo del programa se usa como sinónimo de enojo (INISA, 2016a), los significados son diferentes. Modzelewski (2016) trae a colación a Aristóteles al indicar que es «un apetito penoso de venganza por causa de un desprecio manifestado contra uno mismo o contra los que son próximos, sin que hubiera razón para tal desprecio» (Aristóteles, trad. 1999, pp. 312-313). Esta se dirige, según el autor, hacia personas concretas, merecedoras de este, mas no hacia todos de forma indiscriminada. Es así que el PTGCI (INISA, 2016a) adopta como concepto central y objeto de intervención al enojo, como correlato previo de la ira. En el presente capítulo nos abocamos a la lectura de este a la luz de los conceptos trabajados sobre la educación de las emociones, considerando que dicho protocolo es un aporte favorable en esta dirección.

Al estudiar el dominio emocional y su expresión en la convivencia, destacamos a la autorreflexión como fin de la educación de las emociones en contexto de encierro, en procura de ubicar a la vida humana como fin en sí mismo, dimensionar el valor que reside en cada una de las subjetividades que conforman una sociedad y la atención a que cada una de ellas se desarrolle de la forma más autónoma posible, con sentido cabal de su existencia y de su voluntad frente a lo efímero de los deseos.

La educación de las emociones tiene un gran aporte para hacer a la justicia, entendida como el empoderamiento de todos los ciudadanos de una comunidad política en el análisis de sus metaemociones de forma metódica. Acordamos con Modzelewski (2016, pp. 106-107) al decir que una buena contribución a un programa de educación emocional sería:

a) el desarrollo de la autorreflexión o la metaemoción antes que una emoción en concreto; b) la concentración específica en la adecuación de las emociones a las circunstancias; c) el trabajo sobre el desarrollo de un lenguaje que permita referirse apropiadamente a las emociones; d) el trabajo en comunidad, es decir, en conjunto con otros; e) la integración de las narraciones como herramienta fundamental de este tipo de educación [...]. Las características aquí detalladas ponen el énfasis en un desarrollo de la autorreflexión para que el sujeto pueda evaluar, jerarquizar y priorizar sus propias emociones, cualesquiera que sean, y considere por sus propios medios (y en comunidad con otros) adecuadas o inadecuadas, poniéndolo a salvo, de esta manera, de adoctrinamientos posibles.

El protocolo trabaja con las personas mediante grupos, y con una especial atención sobre el control del enojo como correlato de la ira. Si bien encontramos contradicciones a la hora de denominarlos en función de las sesiones, el mensaje de interponerse a dichos impulsos desde un punto de vista reflexivo, medir las consecuencias de las posibles reacciones - en un contexto donde todo se potencia- e intentar dar cuenta de que el 
enojo es, finalmente, una interpretación de hechos que no tienen una lectura objetiva son aspectos a señalar en la búsqueda del cultivo de la autorreflexión y la generación de voliciones de segundo orden.

No se plantea el estadio óptimo del ser, qué emociones expresar y cuáles no. Se intenta aportar a lo que entendemos como emociones apropiadas; es decir, que las emociones, entendidas como objeto de educabilidad, se correspondan con el contexto en que se manifiestan. No se condena al enojo en sí mismo, sino las formas de manifestarlo y con quiénes, las creencias en torno a él, se dimensiona el potencial riesgo de dejarlo ser. Se trata de adecuar la emoción al medio en que se vive, para poder desarrollarse integralmente en el cultivo de la sana convivencia.

Si consideráramos cuáles serían las formas de medir los resultados subjetivos que el protocolo obtuvo, nos encontraríamos con grandes dificultades. Buena parte de los componentes subjetivos no se encuentran en el plano de la medición. Las acciones pueden ser desacertadas o no, pero no es sobre esa base que podremos saber si la educación tuvo buen cauce. Es la emoción que lleva a la toma de decisiones la que hace a uno sentirse virtuoso y reflexivo, pero lo que se encuentra al alcance del análisis son tan solo las acciones de los sujetos. A ello se agrega que el cauce de las emociones en lo social - y, más aún, sus repercusiones- a veces escapan a la esencia misma de las emociones que lo generan. No se corresponde directamente con el carácter pulsional del placer, sino con la virtud moral que se expresa, muchas veces, a modo de tranquilidad consigo mismo.

Entendemos que cada sujeto que entra en conflicto con la ley merece una adecuación de la respuesta estatal a la su situación concreta. La justicia, entendida como el desistimiento del delito, y la estandarización han demostrado no ir de la mano. En ese sentido, no es disparatado pensar políticas de educación de las emociones que atiendan la situación personal de cada sujeto. Este programa, como señala la evaluación final, no ha sabido atender ese aspecto. Su correlato ha podido cautivar escasamente a la población objetivo.

Las penas de privación de libertad en centros de reclusión del Estado, y especialmente las de los adolescentes, no son, en Uruguay, una vía de intervención que derive en resultados favorables sobre los sujetos ni, por tanto, sobre lo común. Al contrario, funcionan sistemáticamente en el otro sentido. Cuenta de ello dan las ramas académicas de la universidad que producen conocimiento sobre la privación de libertad y sobre sus efectos neurológicos negativos en los adolescentes (Morás, 2012; Bardazano, Corti, Duffau y Trajtenberg, 2015), así como los datos de reincidencia penal (Casa Bertolt Brecht, 2015). Las expectativas de retorno a un mundo de convivencia con el resto de la sociedad se reducen con el paso por las unidades de internación, y ciertas formas impulsivas de responder a los vaivenes emocionales permanecen. La implementación de programas y actividades que les ofrezcan dinámicas habilitantes y reflexivas, que les permitan pensar en sus condiciones de existencia y en sus vínculos con los otros, son espacios 
importantes para minimizar los daños que la privación de libertad y las situaciones previas hayan podido generar en ellos. Sin embargo, el proyecto también se propone como un dispositivo institucional que refuerza emocionalmente en el no lugar a aquellos a quienes caracteriza como «infractores», carentes de numerosas aptitudes que tienen que ver con la condición humana (véanse las páginas 7-10 del PTGCI, INISA, 2016a). El análisis psicosociológico que se hace de los sujetos se corresponde más con valoraciones homogéneas y negativas, sin fundamentación estadística, que con un programa de educación de las emociones que atienda los procesos de cada uno.

Como señalamos al comienzo, el programa que dio origen a la adaptación para el INISA fue pensado y aplicado a una población adulta (Cullen, 1992). La metodología de intervención era enfocada en el sujeto, personalizada e individual, de modo que no se trabajó en las emociones mediante la narración oral con los pares y la puesta en juego de la empatía. Estos dos aspectos marcan la mayor diferencia con el programa abordado en el INISA, cuyo Programa Técnico Psicosocial puso el acento en el trabajo colectivo y la circulación de la palabra. El PTGCI comenzó a implementarse el 20 de junio de 2016, según consta en la evaluación institucional, donde se puntean las principales dificultades analizadas respecto a la implementación. En sintonía con lo trabajando hasta ahora, señalamos algunas de ellas:

1. Hubo tensión entre la implementación del programa y las medidas de seguridad de los centros penitenciarios. Si bien el programa fue elaborado para implementarse con todas las personas privadas de libertad, en algunos centros se llegó a conformar tan solo un grupo de menos de diez participantes. En el CMC - uno de los centros de privación de libertad del INISA — la implementación del programa fue la más atípica. Los grupos no pudieron conformarse debido a la baja disposición de trabajo institucional y a las rispideces existentes entre los adolescentes. Se tuvieron que conformar grupos de un máximo de cinco adolescentes, con los cuales no se pudo trabajar de forma continuada: los cambios de dirección y el predominio de la seguridad determinaron la suspensión de varias sesiones. Se llegó hasta la sesión número 7 (Instituto Nacional de Inclusión Social Adolescente [INISA], 2016b, p. 57).

2. Buena parte de los adolescentes no cuentan ni han contado con políticas educativas de impacto real. No poseen herramientas de lectoescritura básica y tienen poco desarrollado el pensamiento abstracto (INISA, 2016b). Esto dificultó la comprensión de las consignas y un cabal trabajo introspectivo que incidiera en su vida personal.

3. Se superpusieron diversas posturas respecto al enfoque en la implementación. La formación técnica y la experiencia de trabajo grupal de los integrantes de los equipos interdisciplinarios encargados de implementar el programa en 
cada centro fueron un componente que generó disparidad e hizo que un programa pasara a ser varios diferentes en función del lugar de implementación.

4. La tensión existente en todos los centros de privación de libertad estatales, de adolescentes y de adultos, entre seguridad y educación, entre seguridad y espacios comunes, entre seguridad y espacios de desistimiento del delito, no fue ajena a esta experiencia. Agrupar adolescentes fue visto como un riesgo por las direcciones de los centros penitenciarios (INISA, 2016b). Esto deja como corolario el encierro de cada adolescente en su celda por días enteros, con escasas horas semanales de actividad socioeducativa, lo que refuerza la impotencia y las situaciones de tortura física y psicológica.

5. Una vez implementado, el PTGCI se evaluó como «muy extenso» (INISA, 2016b, p. 55). Se encontraron contenidos repetidos en varias actividades y cierta rigidez en los contenidos. Sumado a ello, la discontinuidad de los coordinadores de los grupos de trabajo generó que las dinámicas carecieran de un hilo conductor, lo que fue paliado por los suplentes de la Dirección Nacional de Programas y de las subdirecciones de Programas de los centros.

\section{Consideraciones finales}

Los seres humanos estamos permeados por un conjunto de normas culturales y cívicas que nos permiten vivir en sociedad. Las sociedades las incorporan y transmiten por medio de los sistemas educativos. Se intenta aportar a los sujetos con aquellos componentes que consideramos trascendentes para la convivencia y la felicidad común. Uno de ellos, no del todo desarrollado en los programas educativos, es la educación emocional. En el presente trabajo intentamos plasmar su importancia para la formación integral de los sujetos; esto es, el enaltecimiento de la reflexión y la pausa frente a lo instantáneo del golpe o la violencia verbal cuando emerge un problema intersubjetivo. No buscamos establecer qué emociones son más indicadas, sino qué métodos utilizar para que las emociones a partir de las cuales uno actúa se correspondan con los deseos profundos de las personas, al tiempo que respeten la integridad física y emocional de terceros. La narración y los espacios reflexivos grupales son herramientas importantes de dicha búsqueda.

Los programas de educación emocional se encuentran, entonces, al alcance de ser desarrollados por cualquier sujeto o cualquier institución educativa que así lo considere. La problematización de las emociones por los programas educativos y las instituciones es sustantiva para aportar en la construcción de una sociedad más autorreflexiva. La educación en emociones es necesaria para que los sujetos se encuentren en permanente situación de cambio respecto a sí mismos y convivan mejor. La agencia de los sujetos y la ciudadanía crítica, activa, requieren de dichas emociones para desarrollarse en 
plenitud. El programa educativo estudiado representó un avance en la visibilización de una temática necesaria, como lo es la educación emocional, y más en privación de libertad. Sin embargo, a partir de la evaluación institucional realizada y de la caracterización del contexto en que se mejorar llevó adelante, se identifican carencias institucionales considerables que es preciso mejorar si se quiere tener una incidencia real en la vida de la población objetivo, en pos de mejores condiciones y herramientas emocionales para la vida en sociedad.

\section{Bibliografía}

Aristóteles. (1999). Retórica. Madrid: Gredos.

Bardazano, G., Corti, A., Duffau, N. y Trajtenberg, N. (2015). Discutir la cárcel, pensar la sociedad: Contra el sentido común punitivo. Montevideo: CSIC.

Casa Bertolt Brecht (2015). Medidas no privativas de libertad en adolescentes: Aportes de la Casa Bertolt Brecht. Montevideo: Casa Bertolt Brecht.

Cullen, M. (1992). Cage your rage: An inmate's guide to anger control. Alexandria (VA): American Correctional Association.

Frankfurt, H. (2006). La importancia de lo que nos preocupa: Ensayos filosóficos. Buenos Aires: Katz.

Freud, S. (1930). El malestar en la cultura. Recuperado de http://www.pensamientopenal. com.ar/system/files/2014/12/doctrina38629.pdf.

Goffman, E. (2001). Internados: Ensayos sobre la situación social de los enfermos mentales. Buenos Aires: Amorrortu.

Goleman, D. (1995). Inteligencia emocional. Barcelona: Kairos.

Hegel, G.W.F. (1987). Fenomenología del espíritu. México: Fondo de Cultura Económica. Institución Nacional de Derechos Humanos y Defensoría del Pueblo. (2018). Informe anual a la Asamblea General. Recuperado de https://www.gub.uy/institucion-nacional-derechos-humanos-uruguay/sites/institucion-nacional-derechos-humanos-uruguay/files/documentos/publicaciones/Informe\%20anual\%202018.pdf.

Instituto Nacional de Inclusión Social Adolescente. (2016a). Programa de tratamiento grupal control de la ira Guía de actividades y dinámicas. Manuscrito inédito.

Instituto Nacional de Inclusión Social Adolescente. (2016b). Informe final: Programa control de la ira. Edición 2016. Manuscrito inédito.

Modzelewski, H. (2016). Fundamentos para un programa de educación de las emociones en una sociedad democrática. Andamios, 13(30), 83-110.

Modzelewski, H. (2017). Emociones, educación y democracia: Una proyección de la teoría de las emociones de Martha Nussbaum. México: UNAM. 
Morás, L. E. (2012). Los hijos del Estado: Fundación y crisis del modelo de protección-control de menores en Uruguay. Montevideo: SERPAJ.

Nussbaum, M. (1993). Poetry and the passions: Two stoic views. En J. Brunschwig y M. Nussbaum (eds.), Passions and Perceptions: Studies in Hellenistic philosophy of mind: Proceedings of the Fifth Symposium Hellenisti (pp. 97-149). Cambridge: Cambridge University Press.

Nussbaum, M. (1997). Justicia poética. Barcelona: Andrés Bello.

Nussbaum, M. (2005). Upheavals of thought. Cambridge: Cambridge University Press.

Nussbaum, M. y Sen, A. (1996). La calidad de vida. México: Fondo de Cultura Económica.

Solomon, R. (2007). Ética emocional: Una teoría de las emociones. Barcelona: Paidós.

Sykes, G. (1958). La sociedad de los cautivos: Un estudio de una prisión de máxima seguridad. Princeton: Princenton University Press.

Zaffaroni, E. (1998). En busca de las penas perdidas: Deslegitimación y dogmática jurídico penal. Buenos Aires: Ediar. 